\title{
Uterine Leiomyosarcoma: A Case Report
}

\author{
Forough Forghani ${ }^{1}$, Abolfazl Mehdizadeh Kashi ${ }^{2}$, Kambiz Sadegi $^{3,4}$, Mania Kaveh $^{2,1^{*}}$, Mehrangiz \\ Ghafari $^{5}$
}

\begin{abstract}
Introduction: Uterine leiomyosarcoma (LMS) is a rare cancer originated from smooth muscle lining the walls of the uterus. LMS is known as an aggressive tumor with high mortality and morbidity rates as compared to other uterine cancers, despite the disease stage at the time of diagnosis. In most cases, LMS has been misdiagnosed as a benign uterine leiomyoma following hysterectomy or myomectomy.

Case Presentation: We presented a 53-year-old G7 L7 woman who had referred to GYN clinic in Amir-al-Momenin hospital for abnormal uterine bleeding (AUB) for 6 months. On physical examination, we found an abdominal mass that had grown rapidly in the last 4 months. The computed tomography (CT) scan results showed a heterogeneous mass extending from the epigastric region to the pelvic area. Following an exploratory laparotomy, histopathology report confirmed the diagnosis of LMS. Her uterus, Fallopian tubes and ovaries were removed during a surgery, and she was referred to a gynecologic oncologist for possible chemotherapy. Conclusion: We found that the surgery is the only treatment for LMS; however, there is a little possibility to diagnose LMS before surgery in the patient with uncertain diagnosis and suspicious of LMS. Analysis of LDH and LDH3 levels along with dynamic diethylenetriaminepentaacetic acid (Gd-DTPA) enhanced, and MRI are recommended.

Keywords: Uterine leiomyosarcoma, Gynecologic oncologist
\end{abstract}

\section{Introduction}

Uterine leiomyosarcoma (LMS) is a rare cancer originated from smooth muscle lining the walls of the uterus with a high risk of development and recurrence. LMS is responsible for $8 \%$ of uterine malignancies (1). About $42 \%-60 \%$ of uterine sarcoma cases are related to various subtypes of LMS. Abnormal uterine bleeding (AUB) as well as pelvic pressure, pain and mass are considered as the first findings and symptoms of leiomyoma and sarcomas, which make it difficult to distinguish between them (2-4). LMS is usually diagnosed following a surgery, although in a rare condition, frozen section analysis is used during a surgery. Grade of the tumor and histopathological findings help physicians choose an appropriate clinical behavior and therapeutic approach. There is not a single reliable test to apply before the surgery to distinguish between a benign or malignant uterine disease. We used computed tomography (CT) scan results and histopathology report following an exploratory laparotomy to diagnose LMS in a 53-year-old G7 L7 woman who complained of AUB and rapidly growing abdominal mass. This study was a case report that discussed uterine LMS, the clinical characteristics, diagnosis, outcome, and recent advances.

\section{Case Report}

A 53-year-old G7 L7 woman, living in Zabol, Sistan and
Baluchestan Province, Iran, suffering from AUB for 6 months, was admitted to the emergency room of Zabol hospital, Zabol, Iran, with complaints of heavy vaginal bleeding and passing blood clots in January 2016. She had mild to moderate pain in the lower abdomen toward the epigastric area. The patient noticed a rapidly growing mass in the lower abdomen from 4 months ago. Patient also complained of abdominal bloating and loss of appetite. There was no symptom of painful bladder or bowl syndromes. Furthermore, there was no history of chronic or long-term diseases.

\section{Physical Examination}

On examination, patient looked pale and had stable vital signs with blood pressure (BP) of 120/79 mm Hg, pulse rate $(\mathrm{PR})$ of 96 pulses/second, and respiratory rate (RR) of 18 breaths/minute. On the abdominal examination, a soft to firm, non-tender, mobile mass was palpated in the midline of abdominal area, spreading from lower abdomen to epigastric area. On vaginal examination, there was an enlarged anteverted uterus, like the size of 20 -week pregnancy. There was no cervical motion tenderness (CMT) and no bilateral tenderness on fornix palpation.

\section{Investigation}

The CT scan results showed a heterogeneous mass

\section{Received 13 December 2016, Accepted 20 July 2017, Available online 14 August 2017}

${ }^{1}$ Department of Obstetrics and Gynecology, Zabol University of Medical Sciences, Zabol, Iran. ${ }^{2}$ Endometriosis and Gynecological Disorders Research Center, Iran University of Medical Sciences, Tehran, Iran. ${ }^{3}$ Pain Research Center, Iran University of Medical Sciences, Tehran, Iran. ${ }^{4}$ Department of Anesthesiology, Zabol University of Medical Sciences, Zabol, Iran. ${ }^{5}$ Department of Pathology, Zabol University of Medical Sciences, Zabol, Iran.

*Corresponding Author: Mania Kaveh, Tel: +989123774054, Email: maniakaveh@gmail.com 
with multilocular and multiseptated regions and with approximate size of $93 \times 121 \mathrm{~cm}$, extending from pelvic area to epigastric area. The results also indicated that the mass possibly spread to the uterine body and anterior wall of myometrium while connecting to the uterus.

Due to the pressure of the mass, the ovaries could not be checked. Free fluid was observed in the abdominal cavity. Muscle tissue and bone of hip region were normal and there was no sign of lymphadenopathy. Furthermore, the results of endometrial biopsy, performed on the patient in October 2015, indicated no presence of malignancy. At the same time, the report of Pap smear indicated chronic cervicitis, and in differential blood count, hemoglobin level was 11; therefore, based on these findings and vaginal bleeding from which the patient suffered, a surgery was performed on the patient in January 2016. During surgery, an enlarged uterus, like the size of 20-week pregnancy, was observed, while there was a connection of dense adhesions of the omentum to the mass and anterior abdominal wall. The mass was arisen from the uterine fundus originated from peritoneum and lobulated omentum (Figure 1). The samples from mass along with solid components, cystic and hemorrhage were then sent to the cytology laboratory of the hospital. Uterus, Fallopian tubes and ovaries were removed during the surgery, and the patient received 3 units of packed red blood cells (RBC).

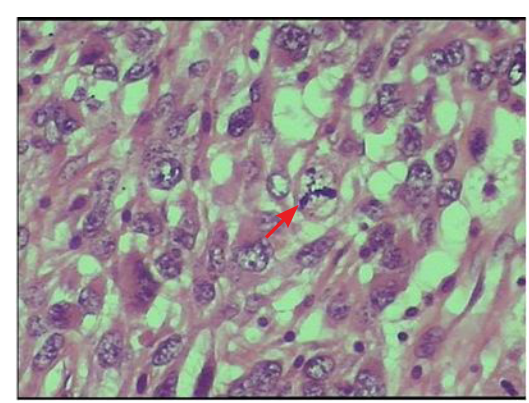

Figure 1. Atypical Mitosis (H\&E staining) $(\times 400)$.
Macroscopic Features of Mass

The dissection of enlarged uterus revealed an oval-shaped mass with a size of $27 \times 20 \times 15 \mathrm{~cm}$ and diameter of $11 \mathrm{~cm}$ inside the uterus cavity that spread throughout the cervix. The cross-section of mass showed a gray-white, fleshy, and lobulated cut surface that contained foci of hemorrhage.

\section{Microscopic Features of Mass}

Evaluation of the tissue sections showed spindle cells containing hyperchromatic nuclei and eosinophilic cytoplasm. The taken images indicated mitotic $(\mathrm{M})$ phase containing atypical mitosis (high-power fields [HPF] $>10 / 10$ ) and foci of necrosis. The total findings suggested LMS with cervix involvement. Peritoneal washings (PW) cytologic analysis indicated no malignant cells.

There was no post-operative complication. The patient was discharged 7 days after the operation in satisfactory condition. After pathology report, the patient was referred to a gynecologic oncologist for possible chemotherapy (Figures 2A-2E).

\section{Discussion}

LMS is considered and is known as an aggressive tumor with high mortality and morbidity $(1,5)$. There is no reliable diagnostic method to distinguish between uterine LMS and benign uterine tumors before surgery. However, due to the increased levels of total lactate dehydrogenase $(\mathrm{LDH})$ and $\mathrm{LDH}$ isoenzyme-3 in the patients with LMS, biochemical evaluations are also considered as a useful method. Among imaging studies, a high vascularity score in Doppler ultrasonography and high signal intensity in diffusion weighted imaging (DWI) also indicate malignant ovarian tumors (6). Diagnosis of LMS and determination of its stage are only based on the histopathology report after the surgery. Surgical staging of LMS includes hysterectomy and bilateral salpingo-oophorectomy (BSO). The tumor size larger than $5 \mathrm{~cm}$ and high mitotic
\end{abstract}
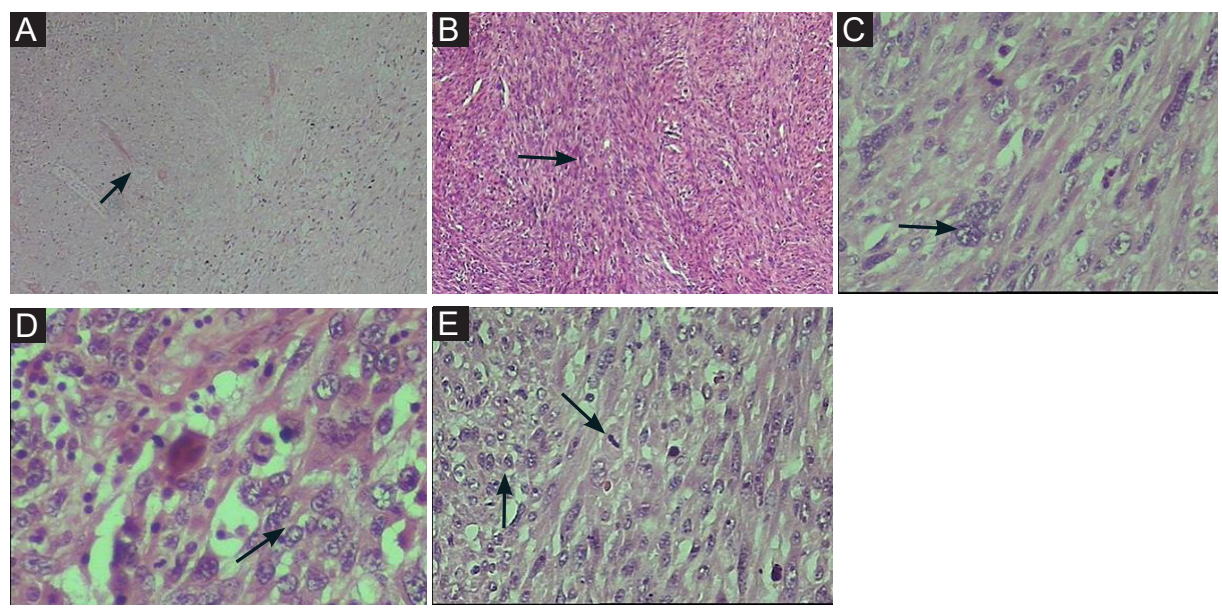

Figure 2. Uterine Leiomyosarcoma. (A) Foci of coagulative tumor cell necrosis (arrow) $(\times 100)$. (B) Spindle cells arranged in intercalating bundle (arrow) (×100). (C) Uterine Leiomyosarcoma (arrow) $(\times 400)$. (D) Pronounced nuclear pleomorphism (arrow) (×400). (E) Nuclear pleomorphism and few mitotic figures (arrow) (H\&E staining) $(\times 400)$. 
index are considered as important prognostic factors (7). Hematogenous spread is the most common form, and lymphatic spread is the rare one. Sarcoma is known as an aggressive tumor, meaning that there is still a high risk of local and distant recurrence after the tumor removal (5). LMS in stages I and II, according to the International Federation of Gynecology and Obstetrics (FIGO) staging systems, has a very high risk of relapse, while its survival rate is also poor after relapse. A study has indicated that the survival rates of LMS I and II are $50 \%$ and $25 \%$, respectively (8). Site of metastasis or recurrence is often around the liver and lungs, which is due to the hematogenous spread (9).The patients with early stage LMS do not really benefit from radiation therapy, while there is no overall survival benefit regarding the radiation therapy. Some prospective studies have also shown the effectiveness of chemotherapy in the treatment of uterine LMS (10). In our case, due to heavy bleeding, we had no time for doing further diagnostic evaluations; therefore, we decided for surgery and considering the LMS-presented macroscopic and microscopic features. Then the patient was referred to a gynecologic oncologist.

\section{Conclusion}

Preoperative algorithm (low risk or high risk) should be attended in order to avoid the occurrence of unexpected LMS, diagnosed on the pathologic post-operative examination and after surgery selection; this should be done in the patients with the history of myoma or the patients highly suspicious to LMS. Clinical, biochemical, and ultrasonographic evaluations, suspicious of LMS, and a pelvic MRI only for highly suspected cases should be performed. We found that surgery is the only treatment for LMS.

\section{Conflict of Interests}

Authors declare that they have no conflict of interests.

\section{Ethical Issues}

Informed consent was obtained from the patient for publishing this case.

\section{Financial Support}

This study was financially supported by Iran University of Medical Science and Endometriosis and Gynecological Disorders Research Center.

\section{References}

1. Brooks SE, Zhan M, Cote T, Baquet CR. Surveillance, epidemiology, and end results analysis of 2677 cases of uterine sarcoma 1989-1999. Gynecol Oncol. 2004;93(1):204208. doi:10.1016/j.ygyno.2003.12.029

2. Baird DD, Dunson DB, Hill MC, Cousins D, Schectman JM. High cumulative incidence of uterine leiomyoma in black and white women: ultrasound evidence. Am J Obstet Gynecol. 2003;188(1):100-107.

3. Buttram VC Jr, Reiter RC. Uterine leiomyomata: etiology, symptomatology, and management. Fertil Steril. 1981;36(4):433-445.

4. Serden SP, Brooks PG. Treatment of abnormal uterine bleeding with the gynecologic resectoscope. J Reprod Med. 1991;36(10):697-699.

5. Kaur K, Kaur P, Kaur A, Singla A. Uterine leiomyosarcoma: A case report. J Midlife Health. 2014;5(4):202-204. doi:10.4103/0976-7800.145175

6. Goto A, Takeuchi S, Sugimura K, Maruo T. Usefulness of Gd-DTPA contrast-enhanced dynamic MRI and serum determination of $\mathrm{LDH}$ and its isozymes in the differential diagnosis of leiomyosarcoma from degenerated leiomyoma of the uterus. Int J Gynecol Cancer. 2002;12(4):354-361.

7. Sagae S, Yamashita K, Ishioka S, et al. Preoperative diagnosis and treatment results in 106 patients with uterine sarcoma in Hokkaido, Japan. Oncology. 2004;67(1):33-39. doi:10.1159/000080283

8. Abeler VM, Royne O, Thoresen S, Danielsen HE, Nesland JM, Kristensen GB. Uterine sarcomas in Norway. A histopathological and prognostic survey of a total population from 1970 to 2000 including 419 patients. Histopathology. 2009;54(3):355-364. doi:10.1111/j.1365-2559.2009.03231.x

9. Wright JD, Seshan VE, Shah M, et al. The role of radiation in improving survival for early-stage carcinosarcoma and leiomyosarcoma. Am J Obstet Gynecol. 2008;199(5):536. e531-538. doi:10.1016/j.ajog.2008.04.019

10. Hensley ML, Wathen JK, Maki RG, et al. Adjuvant therapy for high-grade, uterus-limited leiomyosarcoma: results of a phase 2 trial (SARC 005). Cancer. 2013;119(8):1555-1561. doi:10.1002/cncr.27942

(c) 2018 The Author (s); This is an open-access article distributed under the terms of the Creative Commons Attribution License (http://creativecommons.org/licenses/by/4.0), which permits unrestricted use, distribution, and reproduction in any medium, provided the original work is properly cited. 